\title{
Extremely large epithelial ovarian cancer associated with pregnancy: A case report
}

\author{
VIKTORIA HUSZ, DOROTTYA BUS and GYORGY VAJDA \\ Department of Obstetrics and Gynecology, Zala County Saint Rafael Hospital, H-8900 Zalaegerszeg, Hungary
}

Received April 12, 2017; Accepted September 14, 2017

DOI: $10.3892 / \mathrm{mco} .2017 .1501$

\begin{abstract}
Ovarian malignancies are rare in pregnancy; however, the incidence of abnormal adnexal masses diagnosed during pregnancy is increasing. The most common masses are ovarian cysts, and only 3-6\% of those are malignant. The majority of ovarian masses are diagnosed at an early stage by routine ultrasound examinations. Malignant germ cell tumors are the most common ovarian malignancies associated with pregnancy, while the incidence of epithelial ovarian cancer is only 1:12,000-1:50,000 of pregnancies. The diagnosis and management of ovarian cancer during pregnancy remain unclear due to the rare occurrence and scant data on this condition. We herein report the case of 23-year-old woman with an extremely large ovarian papillary mucinous cystadenocarcinoma diagnosed during pregnancy, identified on ultrasound and magnetic resonance imaging, and treated by surgical resection followed by adjuvant chemotherapy with carboplatin and paclitaxel.
\end{abstract}

\section{Introduction}

Ovarian cancer is the second most frequent gynecological cancer complicating pregnancy $(1,2)$. Due to routine ultrasound examinations, the incidence of abdominal masses diagnosed during pregnancy has increased, and is estimated to be $2-10 \%$ of all pregnancies (1-3). Functional cysts are the most common type of adnexal mass associated with pregnancy $(1,2,4)$. The majority of benign masses are dermoid cysts, serous cystadenomas or mucinous cystadenomas; on rare occasions, endometriomas, hydrosalpinx, heterotopic pregnancy or leiomyomas may be diagnosed (4). Only 3-6\% of all ovarian cysts associated with pregnancy are malignant, and their diagnosis and management is complicated due to the lack of large prospective randomized trials and cohort studies (2). Malignant germ cell tumors are the most common ovarian

Correspondence to: Dr Gyorgy Vajda, Department of Obstetrics and Gynecology, Zala County Saint Rafael Hospital, 1 Zrinyi Street, H-8900 Zalaegerszeg, Hungary

E-mail: drvagy11@gmail.com

Key words: ovarian cancer, pregnancy, magnetic resonance imaging, ultrasound malignancies during pregnancy, while epithelial cancers with low malignant potential are reported less frequently $(1,2,5)$. The incidence of epithelial ovarian cancer is 1:12,000-1:50,000 of pregnancies (1). There are no exact data on mortality due to epithelial ovarian cancer in pregnancy; however, the prognosis is similar to that of non-pregnant patients (6). The rare occurrence and scant data on this condition prompted the reporting of the present case.

\section{Case report}

In September 2016, a 23-year-old nulliparous woman was referred to the Department of Obstetrics and Gynecology of Zala County Saint Rafael Hospital (Zalaegerszeg, Hungary) from another hospital due to suspected preterm labor in the 27 th gestational week. During early pregnancy, a left ovarian mass was incidentally detected, which was considered to be a dermoid cyst. The operation offered was refused by the patient at the time. On bimanual examination, a significantly thinned, one-finger dilated cervix was detected. Corticosteroid prophylaxis was administered and intravenous tocolysis with $\beta$-mimetics was started.

Pelvic ultrasound examination, performed on admission, demonstrated a multicystic ovarian mass $\sim 30 \mathrm{~cm}$ wide, with low-impedance circulation, dislocating the uterus (Fig. 3). The papillary structure of the mass strongly suggested ovarian cancer. Pelvic magnetic resonance imaging (MRI) revealed a bizarre mass in the left ovary measuring 20x26 cm, morphologically and structurally suspicious of malignancy (Figs. 1 and 2). The serum levels of tumor markers were in the normal range, which may be explained by the typical irrelevance of the tumor markers during pregnancy.

Due to the rapid increase in size of the ovarian cyst, an exploratory laparotomy was planned. However, as a result of the preterm rupture of the membranes immediately prior to the operation, the pregnancy could not be preserved; therefore, a cesarean section with left adnexectomy was performed, taking into consideration the age of the patient. During surgery, a large amount of viscous, mucinous fluid was discharged from the cystic mass. Left adnexectomy was performed and biopsies of the right ovary and omentum were collected for histopathological examination (Figs. 4 and 5). A 910-g preterm infant was born in good condition by cesarean delivery.

The histopathological findings confirmed the left ovarian mass to be a low-grade papillary mucinous cystadenocarcinoma 
(Figs. 6 and 7). The results of the biopsies from the omentum and right ovary were negative. The oncological team suggested adjuvant chemotherapy with carboplatin and paclitaxel. According to the decision of the oncological team, 6 cycles of paclitaxel and carboplatin chemotherapy were administered. At the last follow-up visit (May 29, 2017), all the tumor markers were negative, and the control MRI and ultrasound examinations did not reveal tumor recurrence or pathological lymph nodes.

The patient provided informed consent regarding the publication of the case details and associated images.

\section{Discussion}

The incidence of adnexal masses diagnosed during early pregnancy is $1-4 \%$, and the majority are of ovarian origin $(7,8)$. Ovarian tumors rarely cause lower abdominal pain or other symptoms during pregnancy $(1,8)$. Approximately $2-6 \%$ of ovarian tumors associated with pregnancy are malignant $(6,8)$. Ovarian malignant tumors are more frequently reported in primigravidas, and the majority are diagnosed at an early stage ( $<1 \mathrm{C}$ according to the International Federation of Obstetrics and Gynecology staging guidelines) by routine ultrasound examinations (4-6,8-10).

Due to its high sensitivity and specificity for characterizing the morphology of abdominal masses, an ultrasound examination is the optimal diagnostic tool in pregnancy, and it may also differentiate between benign and malignant masses $(7,11)$. The malignant nature of ovarian tumors is indicated by several sonographic characteristics such as size, solid component or complex appearance, papillary structure, internal septations, irregular borders, increased vascularity and low resistance to blood flow (3). Ultrasound examinations are not capable of differentiating between benign and low malignant potential tumors; therefore, further imaging examinations are necessary (7). MRI examinations may be safely performed during the second and third trimesters; furthermore, they may also reveal potential extraovarian spread $(1,11)$.

The high levels of tumor markers are helpful in differentiating between benign and malignant tumors. Carbohydrate antigen-125 is secreted by $80-90 \%$ of epithelial ovarian tumors (3), but in pregnancy the tumor markers may be normally elevated, or may indicate other complications, such as hemolysis/elevated liver enzymes/low platelet count (HELLP syndrome), preeclampsia, or miscarriage (11). The elevation of inhibin, human chorionic gonadotropin and $\alpha$-fetoprotein may indicate germ cell or sex cord stromal ovarian tumors, or fetal aberrations $(3,4)$.

The management of malignant tumors associated with pregnancy represents a challenge, as both the fetal and maternal well-being must be taken into consideration (12). The therapy is guided by the tumor type (histopathological subtype, tumor differentiation, nodal status) and size, gestational age, and the result of the ultrasound examination $(4,5-7,12,13)$. Unilateral cysts sized $<5 \mathrm{~cm}$ that are diagnosed in the first trimester are usually functional cysts $(1,4,10,11)$. Surgical resection is indicated in the case of ovarian tumors during pregnancy that are sized $>6-7 \mathrm{~cm}$, contain a solid component (suggesting malignancy), or are associated with clinical symptoms (such as ascites) $(8,10)$. In the case of asymptomatic ovarian masses,

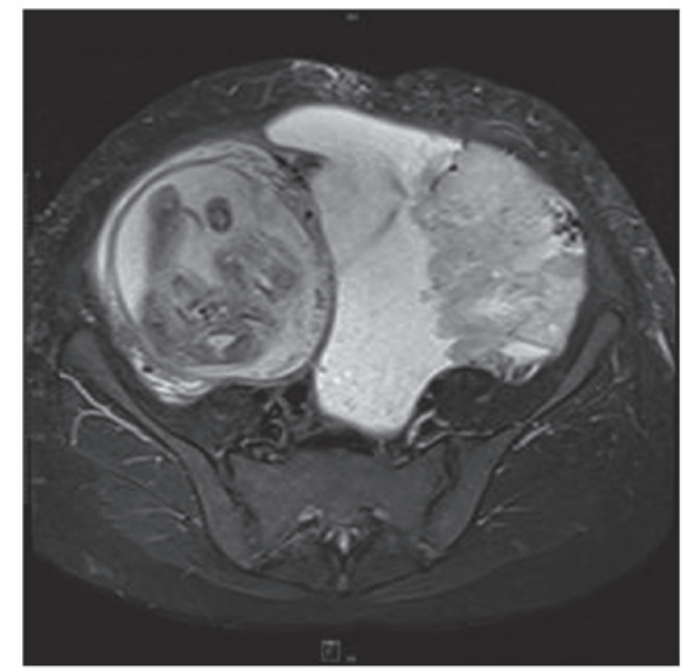

Figure 1. Left ovary, axial short T1 inversion recovery image.

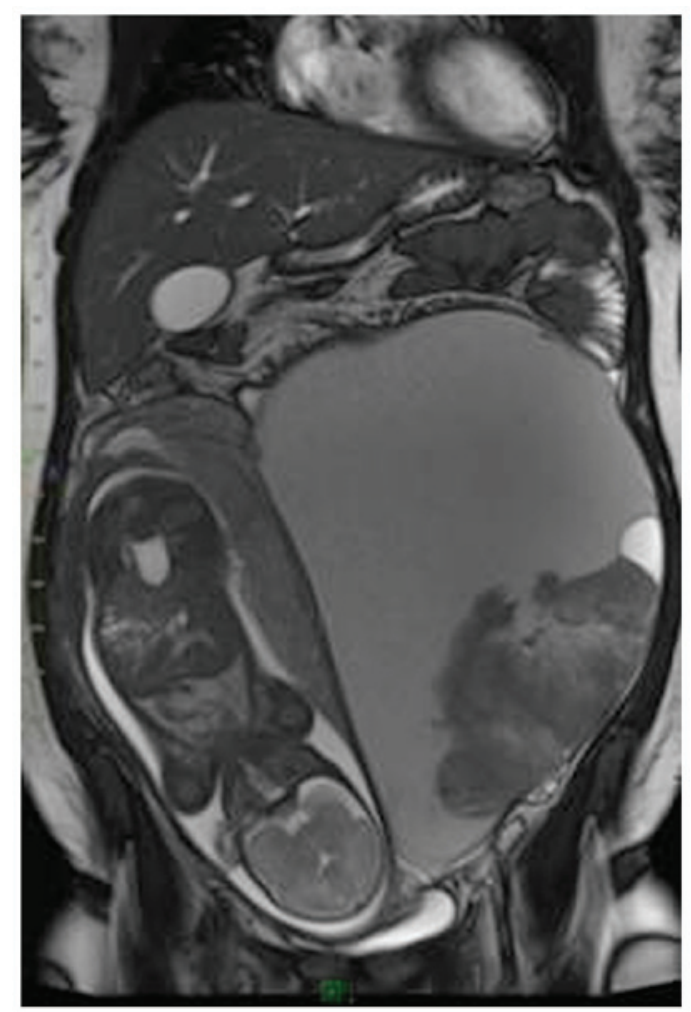

Figure 2. T2 coronal image of left ovary.

the surgery is suggested to be planned between the 16th and 24th gestational week $(6,10,11)$. Due to the unfavorable prognosis of malignant epithelial cancers, the standard therapy is staging laparotomy and adjuvant chemotherapy following debulking surgery (1). In two-thirds of patients diagnosed with epithelial ovarian cancer, there is spread to the peritoneum and lymph nodes; therefore, standard surgery includes the resection of appendages, peritoneal metastases and lymph nodes (13). The majority of the patients with advanced-stage disease require chemotherapy, which should be avoided in the first trimester $(6,13)$. Platinum-based chemotherapy combined with cyclophosphamide may be safely used during 


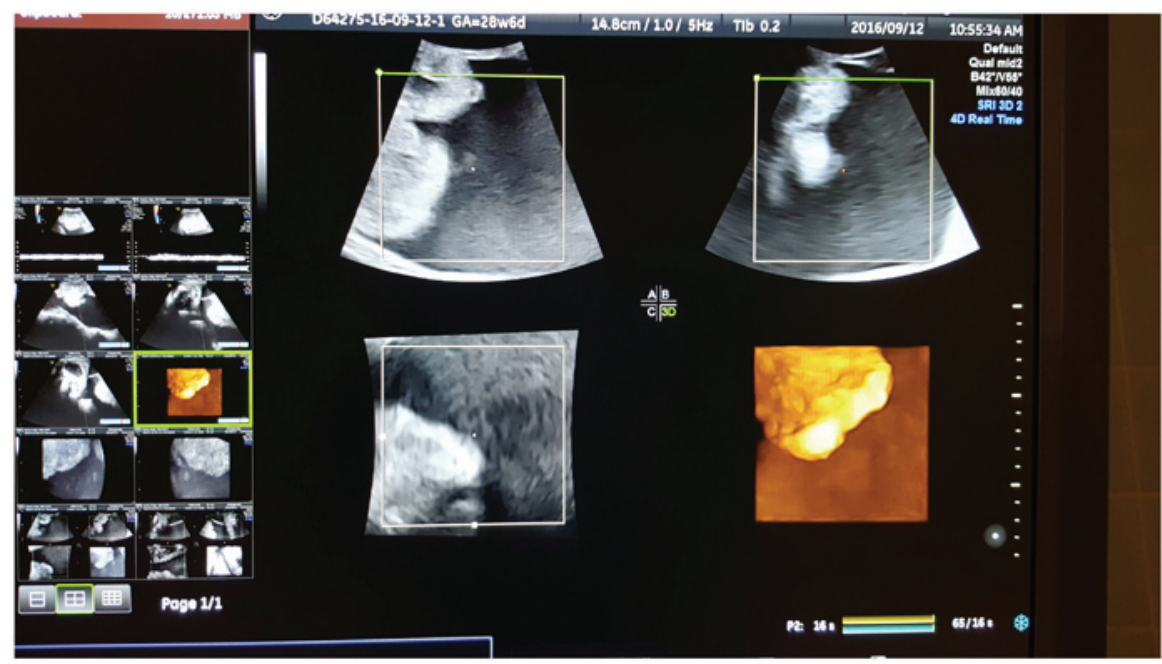

Figure 3. Left ovarian cystic mass on ultrasound examination.

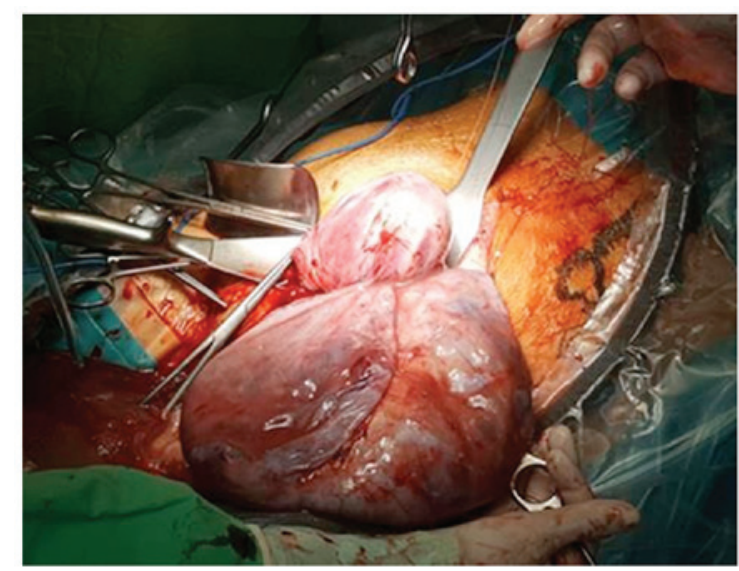

Figure 4. Macroscopic appearance of the left ovary during surgery.

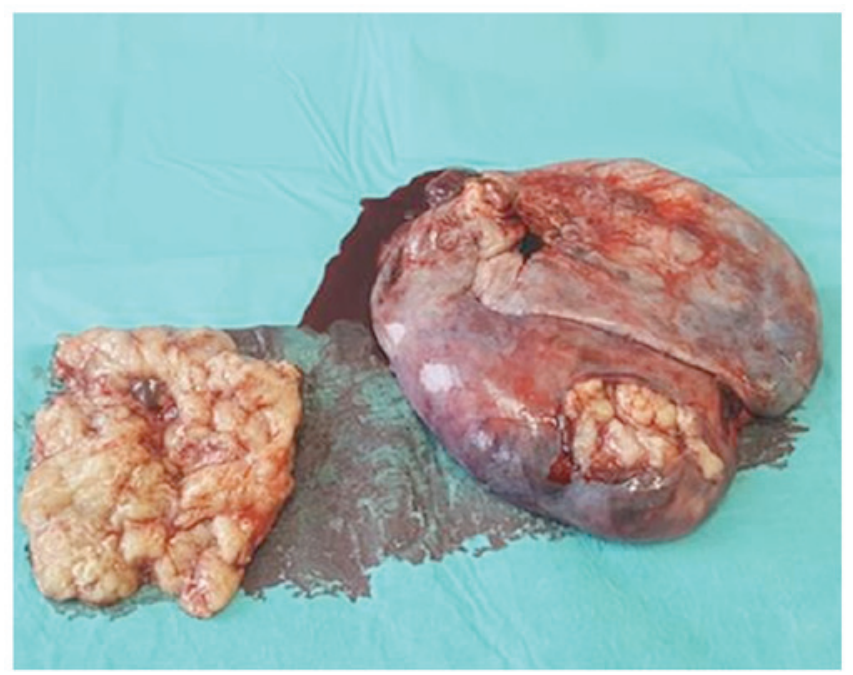

Figure 5. Macroscopic appearance of the resected left ovary.

pregnancy; while the data on the administration of paclitaxel are limited, there has been no report of paclitaxel-induced fetal malformations $(1,6,10)$.

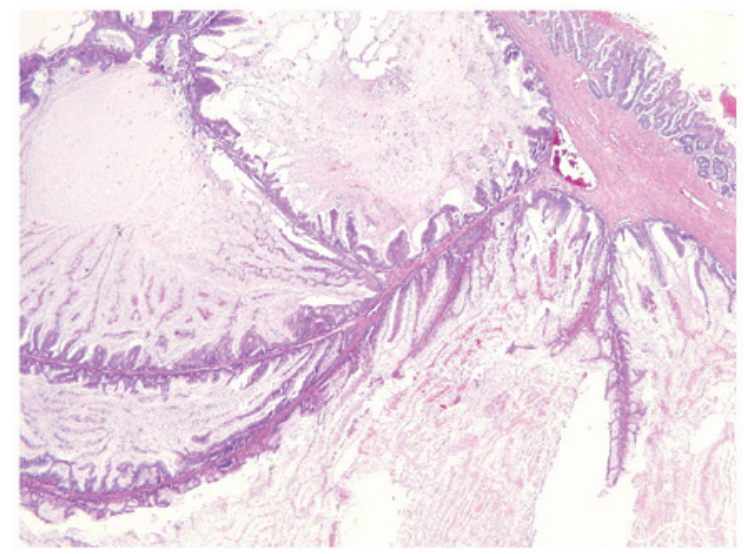

Figure 6. Histological examination of the left ovary under a light microscope (hematoxylin and eosin staining; magnification, x10) revealed low-grade mucinous papillary cystadenocarcinoma pTla, oestrogen and progesterone receptor-negative.

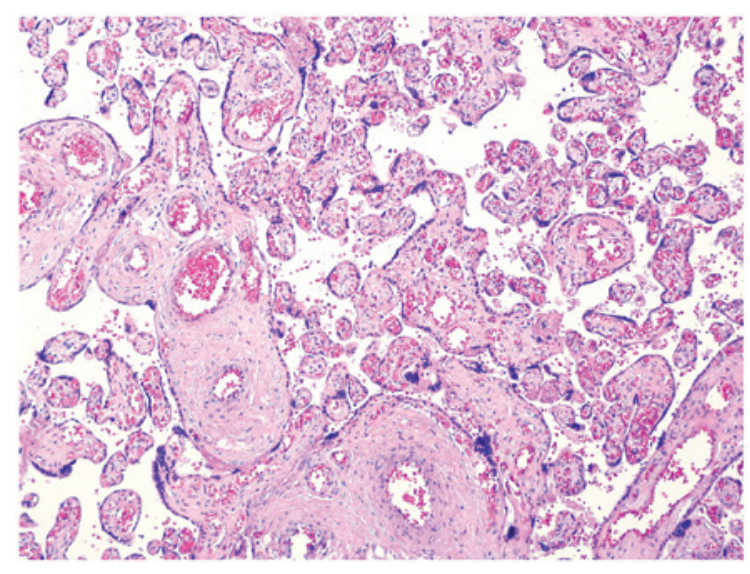

Figure 7. Histological examination of a sample of the normal placenta under a light microscope (hematoxylin and eosin staining; magnification, x10).

In conclusion, malignant tumors associated with pregnancy are becoming more frequent, probably due to the increased age at first pregnancy (12). Although invasive epithelial 
ovarian cancer is a rare diagnosis, maternal mortality is high. The scantiness or relevant studies makes it difficult to select the optimal therapy or diagnostic approach. A multidisciplinary approach involving neonatologists, oncologists and gynecologists appears to be the most important aspect of the management of ovarian cancers during pregnancy $(11,12)$. The outcome of pregnancy is usually favorable; however, the long-term effect of chemotherapy during pregnancy remains unknown.

\section{References}

1. Palmer J, Vatish M and Tidy J: Epithelial ovarian cancer in pregnancy: A review of the literature. BJOG 116: 480-491, 2009.

2. Behtash N, Karimi Zarchi M, Modares Gilani M, Ghaemmaghami F, Mousavi A and Ghotbizadeh F: Ovarian carcinoma associated with pregnancy: A clinicopathologic analysis of 23 cases and review of the literature. BMC Pregnancy Childbirth 8: 3, 2008.

3. Nick AM and Schmeler K: Adnexal masses in pregnancy. Perinatology 1: 13-19, 2010.

4. Yakasai IA and Bappa LA: Diagnosis and Management of Adnexal Masses in Pregnancy. J Surg Tech Case Rep 4: 79-85, 2012.

5. Zhao XY, Huang HF, Lian LJ and Lang JH: Ovarian cancer in pregnancy: A clinicopathologic analysis of 22 cases and review of the literature. Int J Gynecol Cancer 16: 8-15, 2006.

6. Huang HP, Fang CN and Kan YY: Chemotherapy for ovarian mucinous cystadenocarcinoma during pregnancy: A case report. Eur J Gynaecol Oncol 25: 635-636, 2004.

7. Petinga $M$ and Geprgiou $C$ : The management of adnexal masses in pregnant women: A case report and review of literature. Australian Med Stud J, 2015.

8. Takeuchi T, Suzuki S, Hayashi Z, Shinagawa T and Araki T: Primary ovarian tumor undergoing surgical management during pregnancy. J Nippon Med Sch 69: 39-42, 2002.
9. Leiserowitz GS, Xing G, Cress R, Brahmbhatt B, Dalrymple JL and Smith LH: Adnexal masses in pregnancy: How often are they malignant. Gynecol Oncol 101: 315-321, 2006.

10. Roy P, Biswas B, Thomas S, Kumar RM and Jose R: Epithelial ovarian cancer in pregnancy: Report of two cases. Int J Sci Stud 2: 258-261, 2014.

11. de Haan J, Verheecke M and Amant F: Management of ovarian cysts and cancer in pregnancy. Facts Views Vis Obgyn 7: 25-31, 2015.

12. Han SN, Verheecke M, Vandenbroucke T, Gziri MM, Van Calsteren K and Amant F: Management of gynecological cancers during pregnancy. Curr Oncol Rep 16: 415, 2014.

13. Skrzypczyk-Ostaszewicz A and Rubach M: Gynaecological cancers coexisting with pregnancy - a literature review. Contemp Oncol (Pozn) 20: 193-198, 2016. 